\title{
PROPOSAL FOR A SECOND HIPPARCOS
}

\author{
E. $H \varnothing G$ \\ University Observatory \\ 1350 Copenhagen K, Denmark \\ M.S. CHUBEY \\ Pulkovo Observatory \\ 196140 Leningrad, USSR
}

\begin{abstract}
An improved astrometric space mission similar to Hipparcos is proposed for launch ten years after the present Hipparcos. The new mission should provide positions, parallaxes and annual proper motions with an accuracy about one milli-arcsecond for some 400000 stars. The positions from the two missions would give proper motions for the 120000 common stars with an accuracy about 0.2 milli-arcsecond per year, ten times less than expected for the first mission. The improvements of the satellite relate e.g. to: larger telescope aperture, smooth attitude motion, enhanced star mapper for four-colour observations (B V U R) of two million stars. The proposal resulted from discussions with members of the Pulkovo Observatory and the Hipparcos Science Team, and it is being considered by the Mission Control Centre, Moscow.
\end{abstract}

This paper has been submitted for publication in the proceedings of the International Symposium 'Etalon' Satellites held 9-9 June 1991 in Moscow, USSR. 